\title{
FATAL PULMONARY EMBOLISM BY AMNIOTIC FLUID
}

\author{
BY \\ H. D. ATTWOOD \\ From the Department of Pathology of the University of St. Andrews, the Royal Infirmary, Dundee
}

(RECEIVED FOR PUBLICATION JUNE 27, 1955)

Sudden death, during or just after labour, has only too often been attributed to " obstetric shock." Out of this aetiological obscurity, in 1941 and 1942, Steiner and Lushbaugh extracted one recognizable cause of death, the finding in the maternal blood vessels of amniotic material. Recognition of other cases has followed rapidly (see review by Sluder and Lock, 1952), but thus far only three reports have appeared in British journals (Shotton and Taylor, 1949 ; Denniss, Goldie, and Polson, 1954 ; Barry, Geoghegan, and Shea, 1955). The present case is reported with special reference to the techniques that are likely to help the pathologist faced by the problem of death from socalled obstetric shock.

\section{Case Report}

Mrs. M. C., 43 years old, was in her fourth pregnancy, the third having been 19 years before. Her previous pregnancies had been uneventful and had resulted in the spontaneous deliveries of two mature girls and one mature boy.

Her last menstrual period had occurred sometime in December, 1953, and she first attended the antenatal clinic on March 12, 1954 . Thereafter she attended regularly and the examinations gave no cause for alarm. Her urine was always free from sugar and albumin, and her blood pressure was never more than $110 / 80 \mathrm{~mm}$. Hg. Her blood group was $\mathrm{O} \mathrm{Rh}$ positive and on June 6 the haemoglobin was $11.2 \mathrm{~g}$. The Wassermann reaction was negative.

There was a family history of pulmonary tuberculosis, but a chest radiograph in March, 1954, was reported as being within normal limits.

She also gave a history of a pelvic injury, and, as she had a small suprapubic scar, a radiograph of the pelvis was taken. This, too, was reported as being within normal limits.

She was admitted in labour on August 31, pains having begun at 10 a.m.; at 8.30 p.m. her general condition was good (temp. $97^{\circ} \mathrm{F}$., pulse 84-regular in time and force) and blood pressure $130 / 70 \mathrm{~mm}$. $\mathrm{Hg}$. A full-term infant was presenting by the vertex, the head not yet engaged and the membranes intact. Foetal heart sounds were of good tone and regular. Good uterine contractions were occurring at intervals of five to seven minutes. At 9 p.m. she was given $100 \mathrm{mg}$. pethidine intramuscularly.
Satisfactory progress was made, and at 4.20 a.m. (September 1) the foetal head was well into the pelvis and, on rectal examination, the os was considered to be half dilated. As strong uterine contractions were then occurring pethidine was repeated.*

The membranes ruptured spontaneously at 4.45 a.m. and the liquor was seen to be meconium-stained. Ten minutes later the patient felt sick, but did not vomit. She then suddenly lost consciousness and gave several convulsive movements which were considered at the time to be eclamptic in type. She became rapidly cyanotic and dyspnoeic, and died at 5 a.m. At 5.5 a.m., when she was seen by the doctor in charge, no foetal heart sounds could be heard.

\section{Necropsy}

Necropsy was performed six hours after death. The body was that of an asthenic, middle-aged woman of less than average height. The head, neck, and upper thorax were intensely cyanotic and the conjunctivae suffused and injected. The uterus contained a well-formed female infant weighing $3 \mathrm{~kg}$. The head was engaged in the left occipito-anterior position and lay low in the pelvis. The membranes had ruptured, but there was still a considerable amount of deeply meconium-stained liquor in the uterus. The cervix was ragged and dilated to $6 \mathrm{~cm}$. in diameter. No cervical laceration was identifiable. The placenta, which was attached anteriorly, did not come within $8 \mathrm{~cm}$. of the internal os. There was no evidence of placental infarction or separation, or of retroplacental haemorrhage. The uterine wall was congested towards the internal os postexiprly, but there was no evidence of intra-mural haemorrhage or myometrial rupture. The uterine veins were empty.

The maternal thorax was opened under water ; there was no pneumothorax. The pharynx and larynx were clear. The bronchial mucosa was pale and dry. The lungs, which together weighed only $670 \mathrm{~g}$., merely showed some hypostasis. There was no evidence of infarction or of oedema, and the peripheral bronchi were empty.

* Other patients treated with the same batch of pethidine showed no untoward effects. 
The heart $(220 \mathrm{~g}$.) had a greatly dilated but not hypertrophied right side, distended by dark fluid blood, as were the great veins. No air bubbles were seen in the heart's blood. The fossa ovalis was imperforate. The left ventricular myocardium was pale and flabby, but there was no evidence of infarction and the coronary arteries were all patent and free from atheromatous change. The liver $(1,800 \mathrm{~g}$.) showed no evidence of subcapsular or parenchymal haemorrhage. The spleen (180 g.) was congested. The kidneys together weighed 255 g. and had deeply congested medullae. The stellate suprapubic abdominal scar was adherent to the fundus of the bladder, which was otherwise normal. There no evidence of an old pelvic fracture. The adrenals together weighed $10 \mathrm{~g}$. and were normal. The thyroid $(15 \mathrm{~g}$.) was pale and fleshy. The thymus was not identifiable and the pituitary was macroscopically normal. The meninges were healthy. The cerebral veins were markedly congested. The cerebral arteries were free from atheroma and the brain $(1,150 \mathrm{~g}$.) was normal externally and on section. The C.S.F. urea level was $24.5 \mathrm{mg}$. \%.

To summarize, the body was that of a pregnant woman, showing an asphyxial type of cyanosis and a greatly dilated right heart, with a full-term, well-formed infant in an apparently normal uterus.

As no definite diagnosis could be made on the gross finding, blocks were taken for histological examination from the major organs of both mother and child.

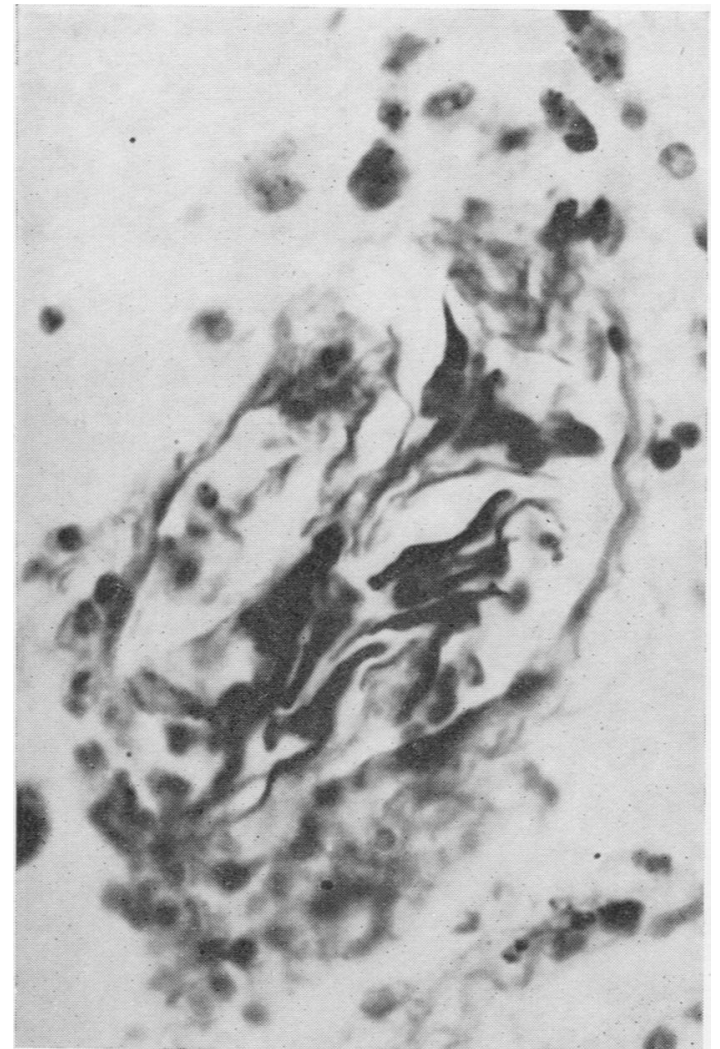

FIG. 1.-Maternal lung. Fuchsinophil squames packing a dilated vessel. Stained picro-Mallory, photographed with minus red (cyan) filter, $\times 720$.

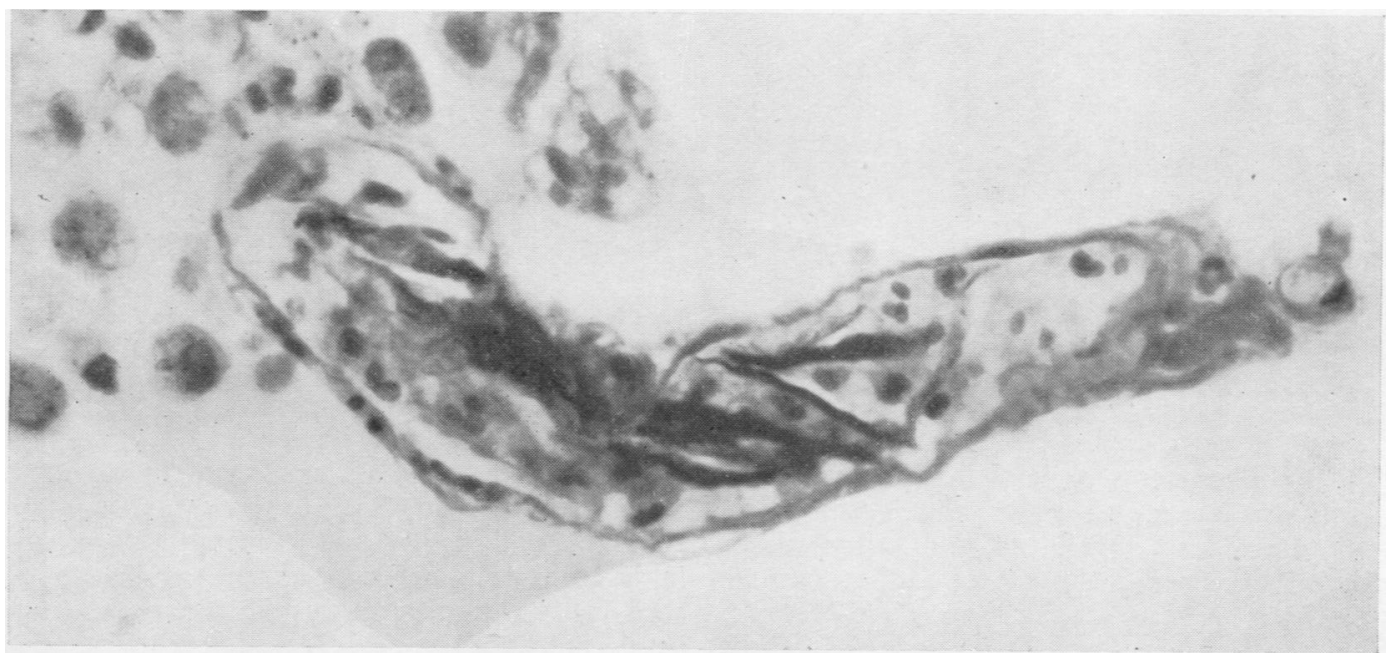

FIG. 1a.-Maternal lung. Alveolar wall with vessel grossly distended by silted fuchsinophil squames. Picro-Mallory, minus red filter, $\times 720$. 
On microscopy, identifiable lesions are seen in the maternal lung, uterus, kidney, brain and heart. The remaining organs examined show no significant lesions.

The lungs show patchy collapse and emphysema, a few areas of alveolar oedema, and many interstitial and intra-alveolar carbon-containing phagocytes. No fibrin thrombi are seen. In all sections (15 blocks taken from random areas of all lobes have been repeatedly sectioned) foreign material is found in the pulmonary arteries and arterioles, and in the alveolar capillaries; all these vessels are dilated. This foreign material consists of the following.

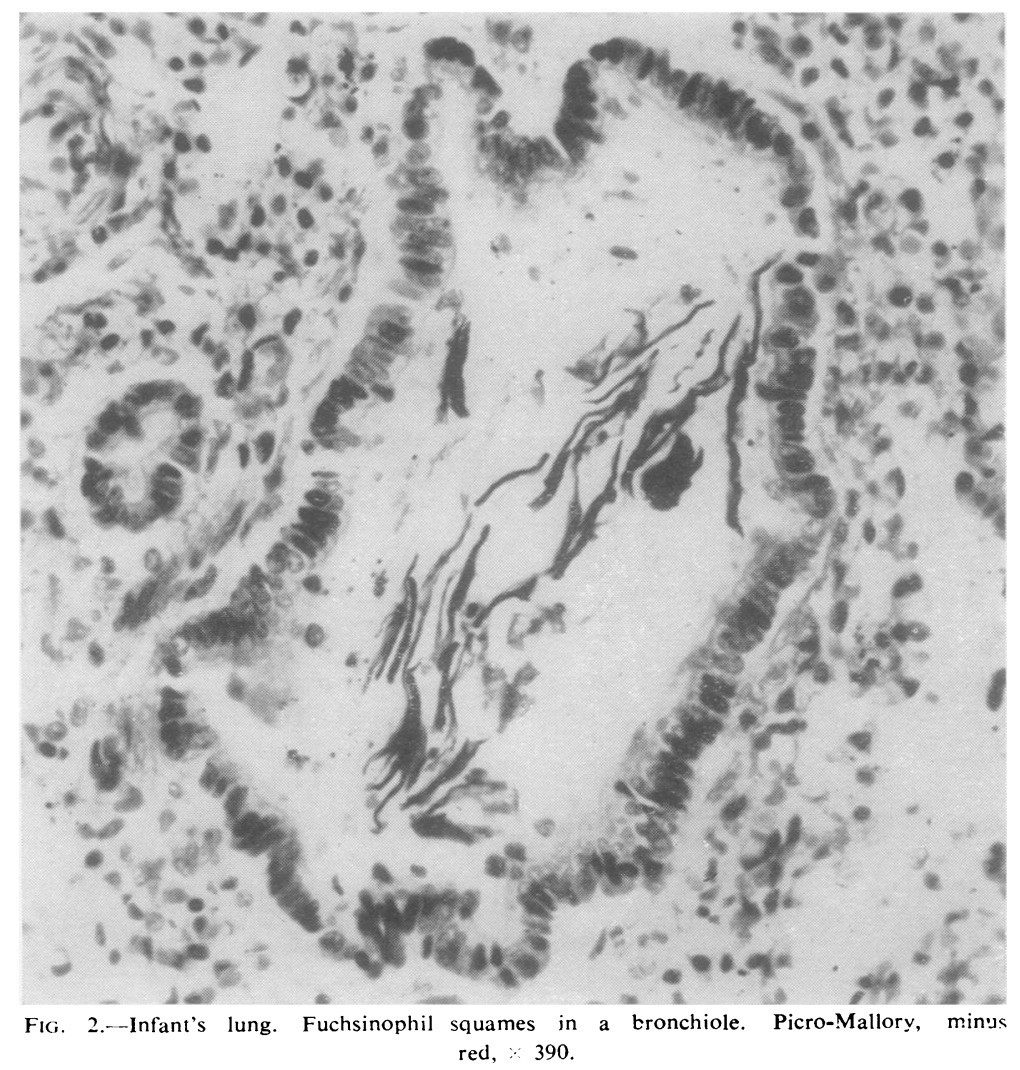
in the vessels. are seen in the infant's lung, in the bronchi, bronchioles (Fig. 2), and in the alveoli, but never

Mucin.-In haematoxylin and eosin preparations, this appears as stringy, amorphous, faintly eosinophilic material in which are enmeshed many polymorphs, often in chains. This "regimentation" of the polymorphs (Fig. 3), although very suggestive, is not diagnostic of mucin. The mucin is strongly metachromatic, and this reaction peisists after incubation with hyalase (Bengers). It stains well with Southgate's mucicarmine, with alcian blue, and with periodic-acid-Schiff (Fig. 4).

Fat.-Tiny globules of Sudanophil material were demonstrated in several of the alveolar capillaries in frozen sections. In at least one paraffin section (Fig. 4) there is a vacuolated appearance suggestive of fat just beyond a globule of mucin plugging a small artery.

Lanugo Hairs. - These are rarely seen, but are easily found by virtue of their anisotropy (Fig. 5). In ordinary light they are seen as somewhat refractile yellowish cylinders streaked with melanin pigment (Fig. 5a). The one illustrated lies amid mucin and blood in an artery and was traced through 10 serial sections.

Bile.-This can be seen quite easily in the haematoxylin and eosin preparations of the infant's lung as golden brown granules lying in the bronchi and in the alveoli. With Stein's iodine test, these granules stain a light green. A few similar granules were found in the mother's lung in an occasional pulmonary arteriole.

Epithelial Squames. - In haematoxylin and eosin preparations these are seen as hyaline eosinophilic plaques, occasionally nucleated, and as basophilic strands, often appearing to silt up a dilated small vessel. They are well shown by picro-Mallory staining as they are strongly fuchsinophil (Figs. 1 and 1a). Exactly similar fuchsinophil squames

Squames and mucin were found in the uterine venous sinuses, and in the glomerular and peritubular capillaries of the kidneys. Surprisingly large collections of squames and mucin are also seen in what appear to be dilated venous sinuses in the kidneys (Fig. 6, 6a, 6b). Several strands of mucin with related polymorphs could be traced in 


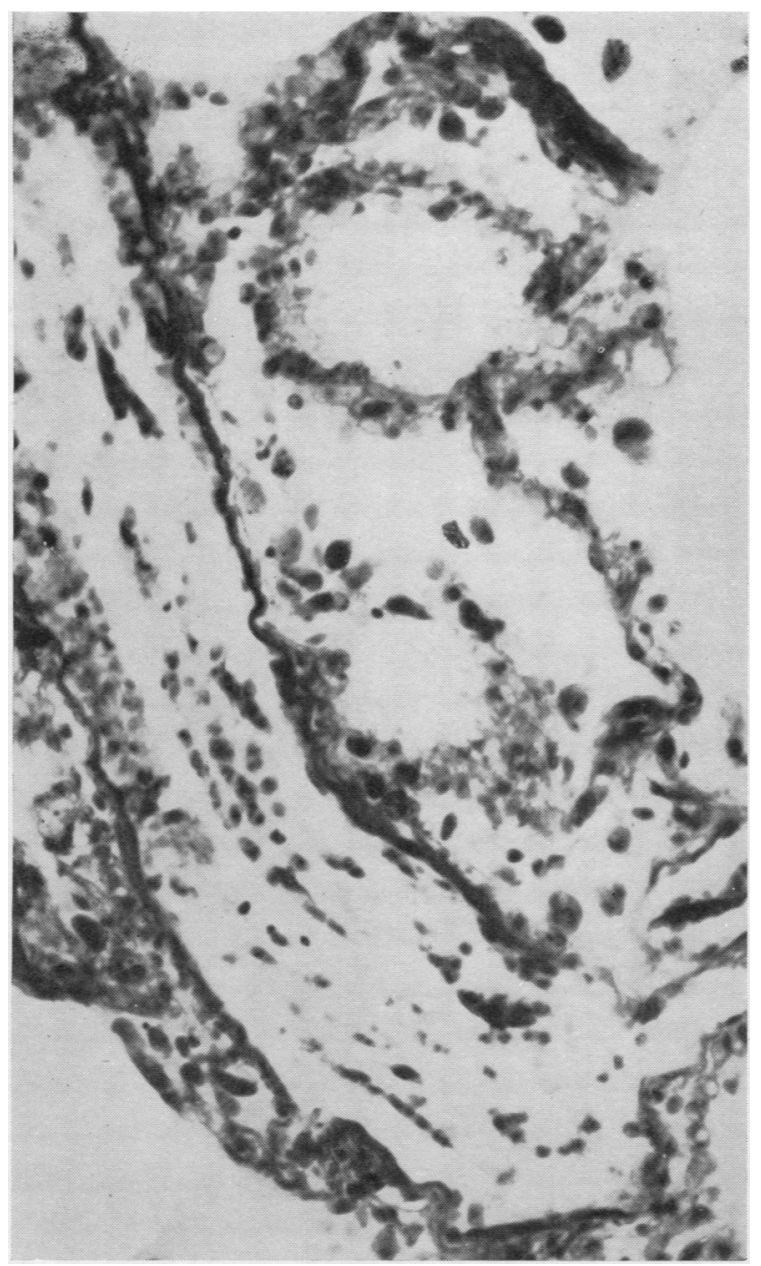

FIG. 3.-Maternal lung. Dilated vessel containing a large amount of mucin (unstained) and a few squames. Note the arrangement of the polymorphs in rows; this is commonly seen where they are enmeshed in mucin. Picro-Mallory, minus red and minus blue, $\times 270$.

serial sections through a capillary in the cerebral basal ganglia. One squame was identified in a capillary deep in the myocardium.

\section{Discussion}

The foreign materials in the pulmonary vessels have been described by earlier writers as epithelial squames, mucin, hair, fat, and bile, identified by their shape and tinctorial or histochemical reactions. Such a collection of foreign material seemed to point to an origin from the amniotic fluid, and the experimental work of Steiner and Lushbaugh (1941), who injected amniotic fluid intravenously into rabbits, seemed to provide adequate confirmation of this. On these grounds it was generally accepted that the material in the maternal vessels was in fact foetal material. However, Tunis (1952) could not accept this, and seems to have considered that the "squames" are in reality maternal in origin and merely loosened endothelial cells. Admittedly, such loosened endothelial cells are not uncommon in post-mortem material, but with the picro-Mallory stain I find that they are blue and never fuchsinophil. Further, the presence of mucin, fat, and hair in a vessel can surely not be explained by any such postulated physiological alteration. That the embolic material had a foetal origin seems certain.

The part of the amniotic fluid that the histologist can most easily recognize in the maternal vessels is the suspended material, mucin, and particulate content. The latter are not present in any great amount in normal amniotic fluid at term; thus, I have rarely found the Wintrobe tube value to be over $5 \%$, but with meconium contamination the content of squames and mucin rises sharply. The mucin is probably entirely derived from the foetal alimentary tract; some of the squames could perhaps be attributed to a stripping action on the skin by bile, although, according to Blystad, Landing, and Smith (1951), there is an increase in the squame and mucin content of meconium itself with increasing maturity of the foetus. It may well be that amniotic fluid becomes more dangerous if it is contaminated with meconium, but there is insufficient information in the reported cases about the degree of meconium staining of the amniotic fluid to allow any valid assessment of this danger. I may add here that a study of 45 sections from the lungs of 21 pregnant women, whose death was not regarded as being due to obstetric shock, failed to reveal any amniotic material.

A possible site of entry of the amniotic fluid into the maternal circulation was not identified in this present case although, at the time of necropsy, it must be admitted that the exact diagnosis was not in mind. In the reported cases the exact site of entry has been defined in some $60 \%$; many of these have been gross lesions involving the placental site, such as ruptured uterus, Caesarean section, placenta praevia, premature separation of the placenta, or even placental infection. With such gross lesions, the amniotic fluid embolism could be merely an associated factor in the causation of death (Tunis, 1952). In other cases the site of entry has been in the region of the cervix, e.g., cervical tears directly related to uterine veins or a cervical tear involving a vessel in a velamentous 


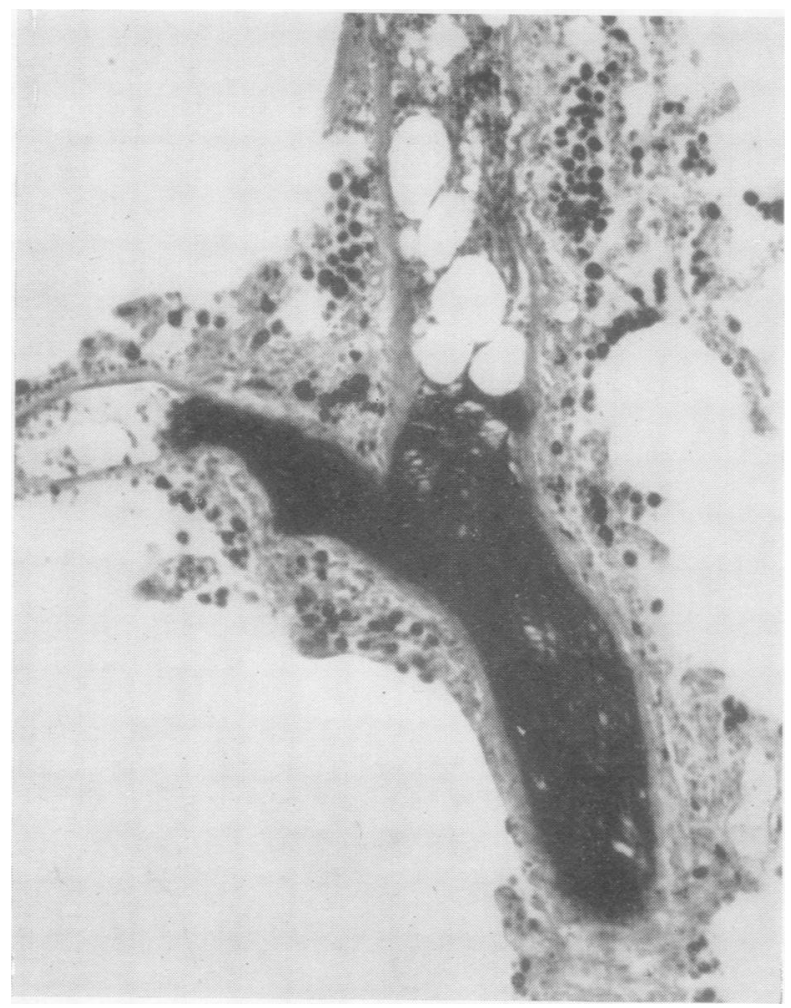

FIG. 4.-Maternal lung. The mucin (black mass) forms a solid plug in this artery; beyond it there are vacuoles suggestive of fat. Periodicacid-Schiff, minus red, 140.

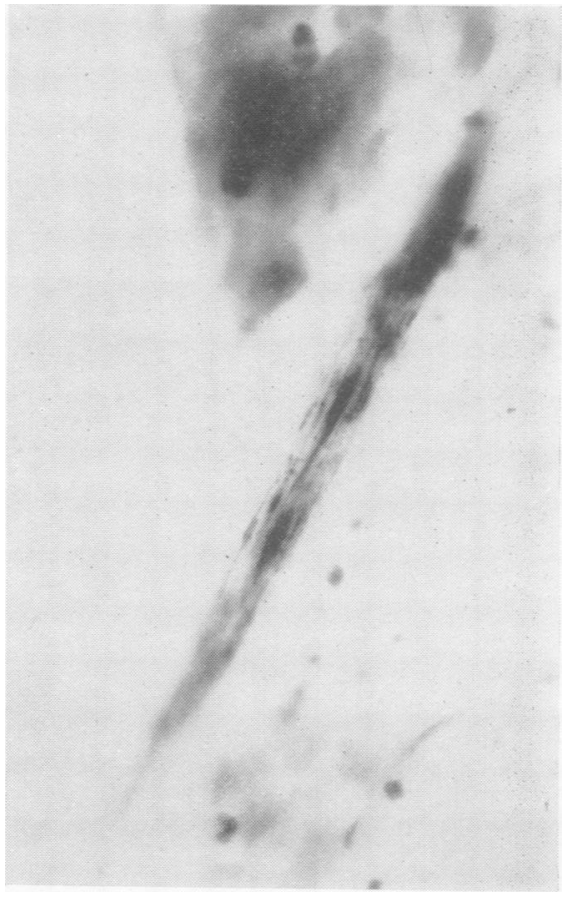

FIG. 5a.-Maternal lung. Pigment-streaked lanugo hair as seen in Fig. 5. Haematoxylin and eosin, 460.

FIG. 5.-Maternal lung. Three anisotropic pieces of a single lanugo hair lying amidst squames and mucin in an artery. Haematoxylin and eosin, polarized light, $\times 160$. 


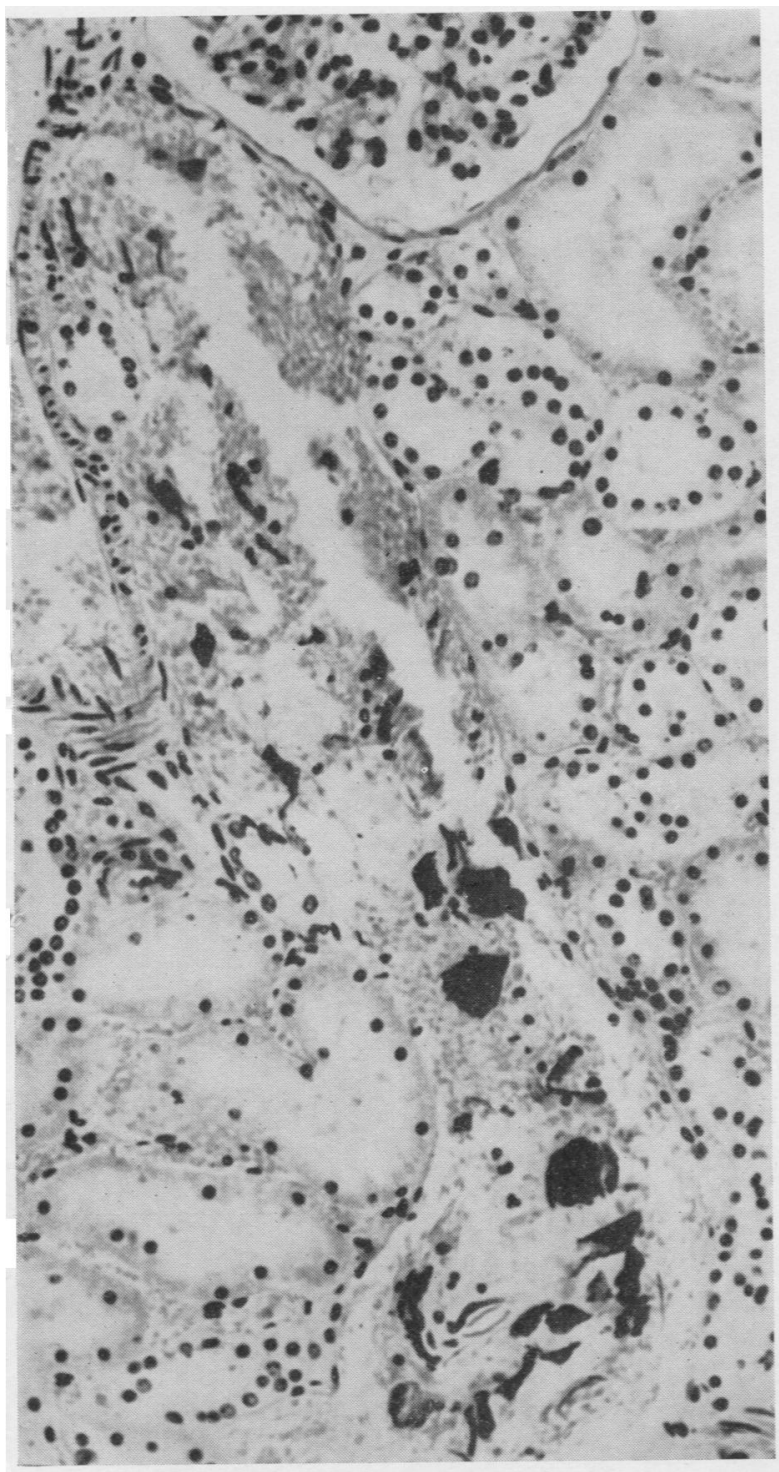

FIG. 6

Fig. 6.-Maternal kidney. Phloxinophil squames are present in this elongated venous channel. The wall of the adjacent artery is seen in the upper left hand corner. The mucin in the vein is unstained by this technique. Haemalum, phloxin, and tartrazine, minus red, $\times 250$

Fig. 6a.-Maternal kidney. Mucin (black) distends this venous channel lying in close relation to an artery. Alcian blue, chlorantine fast red, minus green, $\times 200$.

Fig. 6h.-Maternal kidney. Fuchsinophil squames (dark) lie in the unstained mucin that distends this venous channel. Picro-Mallory, minus red, $\times \mathbf{4 0 0}$.

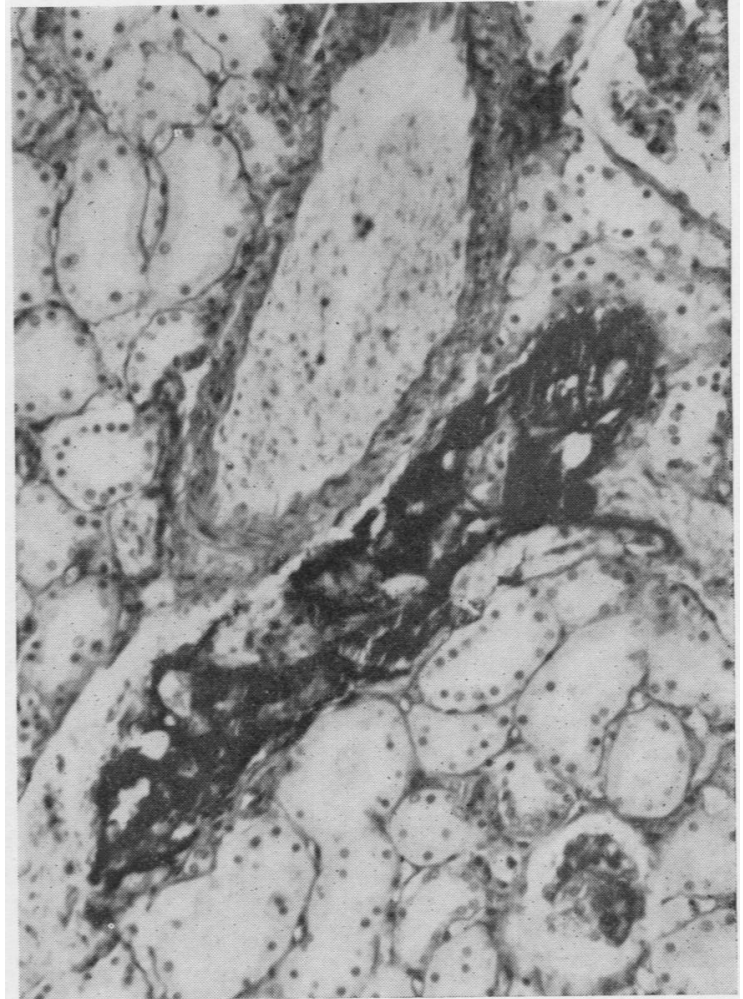

Fig. 6a

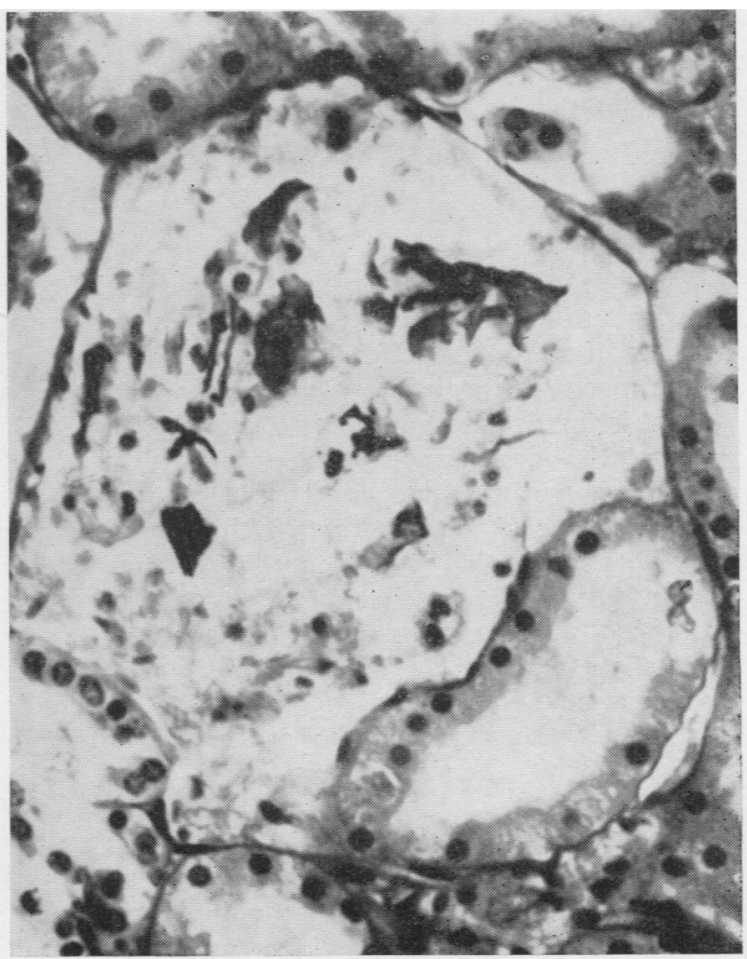

Fig. 6b 
insertion of the cord. The present case certainly showed no such obvious damage to the maternal tissue.

The mechanism underlying the transfer of amniotic fluid was suggested by Steiner and Lushbaugh (1941) to be of the following nature. The membranes having ruptured and the head plugging the outlet, uterine contractions then force fluid from the hind waters into the maternal circulation through some abnormal communication lying above the region of the cephalic tamponade. The amount of embolization would thus be influenced by the strength and frequency of the uterine contractions; this hypothesis is supported by the reported association of amniotic fluid embolism with tetanic uterine contractions and the use of pitocin. The present case accords well with this thesis in that the maternal distress came on almost immediately after the membranes had ruptured, the foetal head being low in the pelvis. The absence of a gross portal of entry and yet very gross and widespread distribution of the foreign material in the lungs makes the use of Reid, Weiner, and Roby's (1953) descriptive term "amniotic fluid infusion" very appropriate in this case. It has to be added, however, that Denniss, Goldie, and Polson have reported amniotic fluid embolism in the absence of labour contractions, and Bowden and Abrahams (1950) reported a case with intact membranes.

That the infusion of amniotic fluid may begin before there is any clinical evidence of rupture of the membranes is suggested by certain of the clinical features both in this present case and in those previously reported. Meconium staining of the liquor in a cephalic presentation is generally accepted as a sign of foetal distress. In many cases of amniotic fluid infusion, including this one, the meconium staining of the liquor has been the first indication that all was not well. Thus, in this case, the first liquor to appear was meconium stained, and at that time there was no evidence of maternal distress, although this appeared very soon afterwards. It is tempting to suggest that the foetus suffers earlier than the mother from the gradually increasing anoxaemia due to the silting up of the maternal pulmonary vessels by the particulate content of the amniotic fluid. That the sudden onset of the shock in the mother is not inconsistent with this hypothesis is suggested by the experimental work of Haggart and Walker (1923) on pulmonary artery occlusion. They showed that no change in the general circulatory state occurred until about 52 to $66 \%$ of the pulmonary artery is obstructed; then a minute increase in the degree of obstruction precipitated a sudden circulatory collapse. The term amniotic fluid infusion could thus still be appropriate.

The particulate content of the amniotic fluid in the pulmonary vessels has been regarded by most writers as being, in itself, harmless, despite the observations of Steiner and Lushbaugh (1941) that filtered amniotic fluid was not lethal in animals. It seems possible that those studying human cases may not have appreciated the true extent of the plugging of the maternal vessels. It cannot be adequately appreciated by a study merely of haemalum-and-eosin-stained sections. Indeed. it was only when sections were stained by the picroMallory method that it was realized how many vessels contained squames, and a study of sections stained by alcian blue, for mucin, and counterstained by the phloxin-tartrazine method, revealed one or more involved vessels in practically every low-power field (16 mm. objective). Further the clinical character of the patient's death closely resembled that produced experimentally by De Takats, Beck, and Fenn (1939) with peripheral pulmonary emboli. The clinical picture, the marked dilatation of the right ventricle seen at necropsy, and the histological findings in the lung all seem to indicate that death was due to blockage of the pulmonary arterial tree.

An anaphylactoid reaction is postulated as the cause of death by many writers, mainly because of the sudden, shock-like character of the seizure. and because many observers thought the histological findings in the lung were not enough to justify a diagnosis of a blocked pulmonary artery. A more specific examination of the lungs might have altered the second argument. The possibility of a hyperergic reaction cannot, however, be lightly dismissed, because the amniotic fluid may carry into the maternal circulation blood group specific substances foreign to the mother.* It has been known since 1930 that if the foetus be a secretor of blood group specific substances the amniotic fluid may contain these in appreciable concentration (Putkonen); that these could act as antigens is clearly shown by the work of Smith (1945), who proved that the rise in isoagglutinin titres occurring in the mothers in heterospecific pregnancies (one in five) was directly related to the secretor status of the infant. Thus amniotic fluid infusion in such a heterospecific pregnancy would be comparable with the sudden infusion of incompatible blood into an already sensitized person. The reaction to incompatible

\footnotetext{
* This idea was suggested to me by Dr. H. G. Morgan, of this Department
} 
blood transfusion is rarely that of a severe anaphylaxis and thus there seems to be no good ground for postulating its presence as the essential factor in this present case. The concurrence of such an incompatibility with amniotic fluid infusion might well alter the clinical picture quite apart from rendering the infusion more certainly lethal.

Haemorrhage has frequently been associated with the less immediately fatal cases of amniotic fluid infusion. In some the haemorrhage has been directly related to gross lesions of the placenta or uterus, but in others there have been associated defects in the blood coagulation mechanism (see papers by Ratnoff and Vosburgh, 1952 ; Reid et al., 1953 ; Schneider, 1950, 1951, 1953, 1955 ; Bowman, 1955). The whole question of coagulation defects in obstetrics has recently received widespread interest (Kennan, 1955 ; Barry et al., 1955 ; Scott, 1955).

The clinical recognition of this condition is not likely to be made with any certainty in the rapidly fatal cases. Cases of "obstetric shock" should perhaps be regarded as due to amniotic fluid infusion if the shock be out of proportion to any haemorrhage that has occurred, and particularly if the labour be one associated with strong contractions, placental abnormalities, or the giving of " pitocin." Since clinical recognition is so difficult, the likelihood of specific treatment is small. It may, however, be reasonable to suggest that, in the absence of haemorrhage sufficient to explain the shock, particularly if shock be accompanied by cyanosis, the administration of oxygen could be usefully coupled with the application of venous tourniquets, as this would lessen the strain on the right ventricle. If death be averted, then attention should be given to the possible onset of haemorrhage associated with coagulation defects. In the treatment of these Scott (1955) suggests that plasma is preferable to "dextran" as a blood volume restorer, as "dextran" may aggravate an already existing hypofibrinogenaemia. Whatever fluid is used considerable clinical acumen is required to estimate the necessary volume, as there seems little doubt that the right side of the heart is under severe stress.

The recognition of this condition at necropsy depends first on the knowledge that amniotic embolism can occur. An early answer may be obtained according to Gross and Benz (1947) by the finding of mucin, fat, or squames in blood from the inferior vena cava or right ventricle. This could be obtained by right ventricular puncture if a full examination was refused. Greasy flecks were noted in blood from the right ventricle by Shotton and Taylor (1949). The absolute diagnosis requires wide histological study, in which the use of a combination of stains such as alcian blue and phloxin may make the diagnosis easier and more efficient. It is only from such specialized examination that the true inc:dence of this condition may be assessed. The published figures of incidence show a quite remarkable variation; thus Steiner and Lushbaugh (1941) estimated the incidence as 1 in 8,000 deliveries, but they later (Steiner, Lushbaugh, and Franks, 1949) amended this, as they had had no further cases in 26,000 deliveries. Gross and Benz (1947) had three maternal deaths in 1,300 deliveries and found all three to be associated with amniotic fluid infusion, whereas Sheehan (1939) in a careful study of 147 maternal deaths could find no example of amniotic fluid infusion. I believe that the use of special staining methods may show that the incidence of this condition is higher than these lower figures suggest.

In conclusion it may be said that amniotic fluid infusion is a histologically defined entity. In a few cases of sudden maternal death the presence of foreign matter of foetal origin in the pulmonary vessels of the mother may be the only lesion found at necropsy. There need be no gross uterine or placental lesion, but an abnormal communication with the maternal circulation must presumably exist; through this amniotic fluid is forced by strong uterine contractions if the head is plugging the pelvic outlet. As death appears to be due to a blockage of the pulmonary vessels by the suspended content of the amniotic fluid, any increase in meconium contamination or in foetal maturity, both known to increase the particulate content of the amniotic fluid, will render the fluid more lethal. It is suggested that an anaphylactic reaction may be an associated lethal factor if the foetus is a secretor in a heterospecific pregnancy. Less severe cases may show haemorrhage due to coagulation defects, the mechanism of which needs further study. As the clinical diagnosis is made largely by exclusion of more easily recognized lesions a therapeutic régime cannot be put forward with any assurance, but in the light of the pathological findings, giving oxygen, reducing venous pressure, and holding in readiness fresh blood and fibrinogen are all reasonable. The pathological diagnosis largely depends, first, on suspicion, based on the knowledge that such a condition as amniotic fluid infusion exists, and thereafter on the determination to demonstrate the 
lesion by scrupulous histological examination of the lungs in all cases of "obstetric shock."

\section{Summary}

The case is described of a 43-year-old woman who died suddenly while in labour. Particulate matter, derived from the amniotic fluid, was demonstrable microscopically in the maternal pulmonary vessels; this was the only lesion found. Such amniotic fluid infusion is discussed mainly from a pathological viewpoint.

My thanks are due to Professor Margaret Fairlie for the clinical notes and for the opportunity to study a series of amniotic fluids, to Professor A. C. Lendrum for his help over all, and to Messrs. Fraser and Corkhill for technical assistance.

\section{REFERENCES} Barry, A. P., Geoghegan, F., and Shea, S. M. (1955). Brit. med. J.,
2, 287 .

Blystad, W., Landing, B. H., and Smith, C. A. (1951). Pediatrics, Bowden, K., and Abrahams, E. (1950). Met. J. Aust. 2, 935.
Bowman, J. A. (1955). Amer. J. Obstet. Gynec., 69, 905.

Denniss, R. G., Goldie, W., and Polson, C. J. (1954). J. Obstet. Gynaec. Brit. Emp., 61, 620.

De Takats, G., Beck, W. C., and Fenn, G. K. (1939). Surgery, 6, 339. Gross, P., and Benz, E. J. (1947). Surg. Gynec. Obstet., 85, 315.

Haggart, G. E., and Walker, A. M. (1923). Arch. Surg. (Chicago), 6,764 .

Kennan, A. L. (1955). Amer. J. med. Sci., 229, 695.

Lushbaugh, C. C., and Steiner, P. E. (1942). Amer. J. Obstet. Gynec., 43, 833 .

Putkonen, T. (1930). Acta Soc. Med. "Duodecim," Ser. A., 14, part 12.

Ratnoff, O. D., and Vosburgh, G. J. (1952). New Engl. J. Med. 247, 970.

Reid, D. E., Weiner, A. E., and Roby, C. C. (1953). Amer. J. Obstet. Gynec., 66, 465.

Schneider, C. L. (1950). In Toxaemias of Pregnancy [Ciba Foundution Symposium], p. 163. Churchill, London.

_- (1951). Surg. Gynec. Obstet., 92, 27.

- (1953). Amer. J. Obstet. Gynec., 65, 245.

- (1955). Ibid., 69, 758.

Scott, J. S. (1955). Brit. med. J., 2, 290.

Sheehan, H. L. (1939). J. Obstet. Gynaec. Brit. Emp., 46, 218.

Shotton, D.IM., and Taylor, C. W. (1949). Ibid., 56, 46.

Sluder, H. M., and Lock, F. R. (1952). Amer. J. Obstet. Gy'nec., 64, 118.

Smith, G. H. (1945). J. Path. Bact., 57, 113.

Steiner, P. E., and Lushbaugh, C. C. (1941). J. Amer. med. Ass., 117,1340 .

-

Tunis, B. (1952). Ibid., 64, 72. 